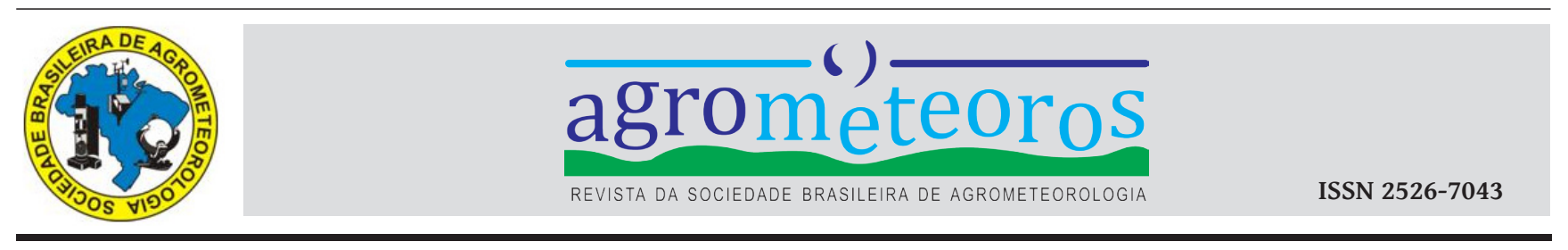

www.sbagro.org.br

\title{
Avaliação dos eventos extremos de precipitação no estado do Piauí
}

\author{
Carlos Antonio Costa dos Santos ${ }^{1(*)}$ e Anna Raquel D. Ramos ${ }^{2}$ \\ ${ }^{1}$ Professor, Unidade Acadêmica de Ciências Atmosféricas, Centro de Tecnologia em Recursos Naturais, Universidade Federal de Campina Grande - \\ UFCG, Av. Aprígio Veloso, 882, Bairro Universitário, Campina Grande - PB. Email: carlos.santos@ufcg.edu.br \\ ${ }^{2}$ Mestre em Meteorologia, Unidade Acadêmica de Geografia, Centro de Humanidades, Universidade Federal de Campina Grande - UFCG, Av. Aprígio \\ Veloso, 882, Bairro Universitário, Campina Grande - PB. Email: anna.raquelramos@gmail.com \\ ${ }^{(*)}$ Autor para correspondência
}

\section{INFORMAÇÕES}

\section{História do artigo:}

Recebido em 16 de Junho de 2017

Aceito em 10 de agosto de 2017

\section{Termos para indexação:}

desertificação

El Niño

uso da terra

temperatura da superfície do mar
RESUMO

O presente estudo verificou a dinâmica espaço-temporal dos índices de extremos climáticos dependentes da precipitação diária no estado do Piauí, correlacionando-os com o uso e ocupação do solo, assim como, com as anomalias de temperatura dos Oceanos Atlântico e Pacífico. Foram utilizadas 34 imagens do sensor TM do satélite Landsat-5 e dados diários de precipitação, oriundos do Instituto Nacional de Meteorologia (INMET), de treze estações meteorológicas, além de dados das anomalias de temperatura da superfície dos Oceanos Tropicais para as regiões escolhidas. Foi observado diminuição da precipitação total anual no Estado do Piauí. Verificou-se que o regime de chuvas na porção norte do Estado sofre influência das anomalias de TSM do Pacífico e do Atlântico. Os índices que representam os extremos de chuva apresentaram correlações, com significância estatística, com as regiões de Niño 1+2 e Niño 3, Niño 3.4, Niño 4 no Pacífico e com a região do Atlântico Sul Tropical. Logo, há forte influência do ENOS e do Dipolo do Atlântico sobre o clima do Piauí. No entanto, a parte sul do Estado apresentou poucos resultados com significância estatística sugerindo que o regime de chuvas nessa região pode ser mais influenciado por sistemas locais.

(c) 2017 SBAgro. Todos os direitos reservados.

\section{Introdução}

Tendências e mudanças nos extremos de precipitação e temperatura têm sido focos de pesquisas ao longo das últimas décadas. A Equipe de Peritos sobre Índices de Detecção de Mudanças Climáticas (ETCCDMI), observando essa problemática, criou um conjunto de índices de extremos climáticos dependentes de dados de precipitação e temperatura para permitir a uniformidade na análise de eventos climáticos extremos em diferentes regiões em todo o mundo (VILLAFUERTE II et al., 2014). Os trabalhos pionei- ros na aplicação destes índices para diferentes regiões foram Zhang et al. (2005), Klein Tank et al. (2006), New et al. (2006), Haylock et al. (2006) e Alexander et al. (2006) todos oriundos de workshops desenvolvidos com dados e pesquisadores de diferentes países.

Segundo Guerreiro et al. (2013), os recursos hídricos são de grande importância para muitas atividades econômicas, sendo essencial para a agricultura, abastecimento de água, indústria e produção de energia. Oyama e Nobre (2004) afirmam que na área semiárida do NEB, a precipitação anual é, em média, inferior a $800 \mathrm{~mm}$, apresentando 
DISTRIBUICÃO ESPACIAL DAS 13 ESTAÇÕES UTILIZADAS

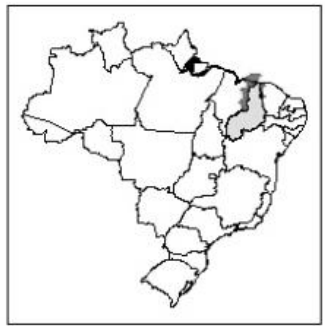

a)

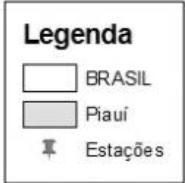

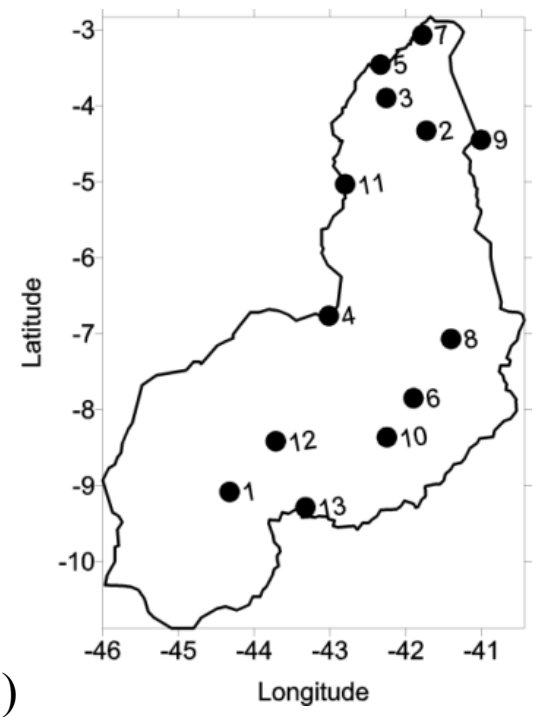

Figura 1. Localização do estado do Piauí (a) e distribuição espacial das estações meteorológicas utilizadas nesse estudo no referido Estado (b).

alta variabilidade interanual.

No Nordeste do Brasil (NEB) existem grandes áreas de terras degradadas submetidas a processos de desertificação. Essas áreas degradadas são resultado, principalmente, de antigas práticas agrícolas (agricultura de subsistência), queima da biomassa e manejo inadequado do solo. Esse problema é de grande importância social, pois nessa região se encontra cerca de $30 \%$ da população do Brasil, no entanto, existem poucas informações disponíveis sobre a relação entre os índices de extremos climáticos e o uso e ocupação do solo nessas regiões (Almeida-Filho e Carvalho, 2010).

Nesse sentido, o presente estudo pretende verificar a dinâmica espaço-temporal dos índices de extremos climáticos dependentes da precipitação pluvial diária no estado do Piauí e correlacionar com o uso e ocupação do solo, assim como, com as anomalias de temperatura dos Oceanos Atlântico e Pacífico, visto que a variabilidade anual e mensal da precipitação no NEB é altamente relacionada com diferentes sistemas atmosféricos, que estão ligados à temperatura da superfície do mar (TSM) destes oceanos tropicais (Hastenrath e Greischar, 1993, Uvo et al, 1998 e Hastenrath, 2012).

\section{Material e métodos}

\section{Localização da área de estudo e dados}

O Estado do Piauí está localizado no NEB (Figura 1a) e, segundo o IBGE (2012), possui uma área de aproximadamente $251.576,644 \mathrm{~km}^{2}$, tem uma população de 3.118 .360 habitantes. É o terceiro maior Estado do Nordeste, perdendo em área apenas para a Bahia e o Maranhão.

Foram utilizadas 34 imagens do sensor TM do satélite Landsat- 5 correspondente às órbitas 217, 218, 219, 220, 221 e pontos $062,063,064,065,066,067$. Cada órbita/ponto foi representada por duas imagens de períodos distintos (1990 e 2000), sendo possível fazer um estudo temporal do uso da terra. As imagens foram obtidas no website do Instituto Nacional de Pesquisas Espaciais (INPE).

Os dados diários de precipitação foram oriundos do Instituto Nacional de Meteorologia (INMET). Foram escolhidos os dados de treze estações meteorológicas que podem ser visualizadas na Figura $1 \mathrm{~b}$ e descritas na Tabela 1 , as quais estão bem distribuídas espacialmente dentro do Estado. Os dados das anomalias de temperatura dos Oceanos Tropicais, para todas as regiões de Niño, TNAI (Tropical Atlantic Northern Index) e TSAI (Tropical Atlantic Southern Index) foram obtidos através do website da NOAA (https:// www.esrl.noaa.gov/psd/data/climateindices/list/).

\section{Metodologia}

O software utilizado na análise do uso e ocupação das terras através das imagens TM Landsat -5 foi o Spring 5.1.8, e foi escolhido por ser um banco de dados geográfico de $2^{\circ}$ geração, desenvolvido pelo INPE para ambientes UNIX e Windows. A partir das imagens corrigidas geometricamente foram geradas no módulo LEGAL do Spring as imagens do NDVI (Índice de Vegetação por Diferença Normalizada) para os dois períodos estudados, ou seja, 1990 e 2000. Para uma melhor interpretação dos resultados do NDVI foi realizado um fatiamento em seis classes. O fatiamento consistiu em definir o agrupamento dos níveis de cinzas. A classificação das imagens consistiu em associar determinados níveis de cinzas a classes específicas predefinidas no momento da criação do banco de dados. A definição das classes é mostrada na Tabela 2.

Para a obtenção dos índices de extremos climáticos de- 
Tabela 1. Descrição das estações meteorológicas estudadas no Estado do Piauí.

\begin{tabular}{lcccc}
\hline \multicolumn{1}{c}{ Estações } & ID & Latitude & Longitude & Altitude (m) \\
\hline Bom Jesus do Piauí & 1 & $-44,326$ & $-9,083$ & 332 \\
Caldeirão & 2 & $-41,733$ & $-4,330$ & 160 \\
Esperantina & 3 & $-42,259$ & $-3,899$ & 61 \\
Floriano & 4 & $-43,017$ & $-6,767$ & 150 \\
Luzilândia & 5 & $-42,366$ & $-3,466$ & 20 \\
Morro dos Cavalos & 6 & $-41,900$ & $-7,850$ & 242 \\
Parnaíba & 7 & $-41,783$ & $-3,069$ & 57 \\
Picos & 8 & $-41,404$ & $-7,071$ & 208 \\
Piriri & 9 & $-41,010$ & $-4,450$ & 161 \\
São João do Piauí & 10 & $-42,251$ & $-8,365$ & 235 \\
Teresina & 11 & $-42,801$ & $-5,035$ & 74 \\
Vale do Gurgueia & 12 & $-43,717$ & $-8,417$ & 265 \\
Caracol & 13 & $-43,324$ & $-9,286$ & 523 \\
\hline
\end{tabular}

Tabela 2. Quantificação das classes de cobertura do solo para o Estado do Piauí.

\begin{tabular}{lcccc}
\hline Cobertura do solo & \multicolumn{2}{c}{$\left(\mathbf{k m}^{\mathbf{2}}\right)$} & \multicolumn{2}{c}{$\mathbf{( \% )}$} \\
\hline & 1990 & 2000 & 1990 & 2000 \\
\cline { 2 - 5 } Água & 622,52 & 763,59 & 0,25 & 0,30 \\
Mata & $120.379,04$ & $119.563,24$ & 47,80 & 47,47 \\
Transição & $62.466,39$ & $67.842,66$ & 24,80 & 26,94 \\
Culturas & $1.315,27$ & $5.556,92$ & 0,52 & 2,21 \\
Solo exposto & $64.716,58$ & $56.881,48$ & 25,70 & 22,58 \\
Outros & $2.359,11$ & $1.250,76$ & 0,94 & 0,50 \\
\hline
\end{tabular}

pendentes da precipitação foi utilizado o RClimdex que é um software baseado no programa de linguagem $\mathrm{R}$ com saídas de tabelas e figuras e tem capacidade de calcular 27 índices básicos recomendados pelo CCI/CLIVAR "Climate Change Action Monitoring and Índices" (ETCCDMI), bem como outros índices de temperatura e precipitação (Zhang e Yang, 2004). O mesmo tem sido bem utilizado, em vários estudos como os de Haylock et al. (2006) na América do Sul, Santos e Brito (2007) no Semiárido Brasileiro e Santos et al. (2011) em Utah, Estados Unidos da América, e é adquirido de forma gratuita através de download no website do ETCCDMI (http://etccdi.pacificclimate.org/data.shtml).

Os 8 (oito) índices de extremos climáticos derivados de dados de precipitação diária, utilizados nessa pesquisa por se adequarem às condições climáticas da região, estão descritos na Tabela 3. Na avaliação da homogeneização dos dados e no cálculo dos índices foi utilizada a metodologia de Zhang et al. (2005) implementada no software RClimdex. O controle de qualidade, que é um pré-requisito para a obtenção dos índices de extremos climáticos, consistiu na identificação de erros nos dados, conforme descrito por Viney e Bates (2004).

O software fornece dados estatísticos, para todos os índices, tais como: tendência linear anual calculada pelo método de mínimos quadrados; nível de significância estatística da tendência (valor $p$ ); coeficiente de determinação $\left(r^{2}\right)$ e erro padrão de estimativa; assim como os gráficos das séries anuais. Como os dados não se ajustaram a uma distribuição de frequência normal, foi utilizado o teste não-paramétrico de Mann-Kendall (Sneyers, 1990) para identificar se as ten- 
dências apresentam nível de significância para o intervalo definido. Nesse estudo só foram consideradas significativas às tendências, que apresentam nível de significância igual ou superior a $90 \%(\mathrm{p} \leq 0,1)$, conforme mostrado na Tabela 4 .

O método de correlação de Spearman $(\rho)$, ao nível de significância de $5 \%(\mathrm{p}<0,05)$, foi utilizado na análise das relações existentes entre as anomalias anuais de TSM dos Oceanos Pacífico (regiões de Niño 1+2, Niño 3, Niño 3.4 e
Niño 4) e Atlântico (TNAI e TSAI) (Menezes et al., 2008), no período de 1971 a 2010, e os índices de extremos climáticos anuais, obtidos para o Estado do Piauí. O coeficiente de correlação de Spearman é uma medida de correlação não-paramétrica. Ao contrário do coeficiente de correlação de Pearson não requer suposição de linearidade entre as variáveis. Deste modo, este coeficiente não é sensível a assimetrias na distribuição, nem à presença de outliers, não

Tabela 3. Índices climáticos dependentes da precipitação pluvial diária, com suas definições e unidades. O RR é o valor da precipitação diária. $R R \geq 1 \mathrm{~mm}$ representa um dia úmido e $\mathrm{RR}<1 \mathrm{~mm}$, um dia seco.

\begin{tabular}{|c|c|c|c|}
\hline ID & Nome do Indicador & Definição & Unidade \\
\hline DCS & Dias consecutivos secos & $\begin{array}{l}\text { Número máximo de dias } \\
\text { consecutivos com } \mathrm{RR}<1 \mathrm{~mm}\end{array}$ & dias \\
\hline DCU & Dias consecutivos úmidos & $\begin{array}{l}\text { Número máximo de dias } \\
\text { consecutivos com } R R \geq 1 \mathrm{~mm}\end{array}$ & dias \\
\hline Rx1dia & $\begin{array}{l}\text { Quantidade máxima de precipitação em } \\
\text { um dia }\end{array}$ & $\begin{array}{l}\text { Máximo anual de precipitação } \\
\text { em } 1 \text { dia }\end{array}$ & $\mathrm{mm}$ \\
\hline Rx5dias & $\begin{array}{l}\text { Quantidade máxima de precipitação em } \\
\text { cinco dias }\end{array}$ & $\begin{array}{l}\text { Máximo anual de precipitação } \\
\text { em } 5 \text { dias consecutivos }\end{array}$ & $\mathrm{mm}$ \\
\hline $\mathrm{R} 10 \mathrm{~mm}$ & $\begin{array}{l}\text { Número de dias com precipitação acima } \\
\text { de } 10 \mathrm{~mm}\end{array}$ & $\begin{array}{l}\text { Número de dias em } 1 \text { ano em } \\
\text { que a precipitação foi } \geq 10 \mathrm{~mm}\end{array}$ & dias \\
\hline $\mathrm{R} 20 \mathrm{~mm}$ & $\begin{array}{l}\text { Número de dias com precipitação acima } \\
\text { de } 20 \mathrm{~mm}\end{array}$ & $\begin{array}{l}\text { Número de dias em } 1 \text { ano em } \\
\text { que a precipitação foi } \geq 20 \mathrm{~mm}\end{array}$ & dias \\
\hline R95p & Dias muito úmidos & $\begin{array}{l}\text { Soma total da precipitação em } \\
\text { um ano de } 5 \% \text { dos dias mais } \\
\text { chuvosos }\end{array}$ & dias \\
\hline R99p & Dias extremamente úmidos & $\begin{array}{l}\text { Soma total da precipitação em } \\
\text { um ano de } 1 \% \text { dos dias mais } \\
\text { chuvosos }\end{array}$ & dias \\
\hline $\mathrm{R} 50 \mathrm{~mm}$ & $\begin{array}{l}\text { Número de dias do ano com precipitação } \\
\text { superior a } 50 \mathrm{~mm} / \text { dia }\end{array}$ & $\begin{array}{l}\text { Número de dias em } 1 \text { ano em } \\
\text { que a precipitação foi } \geq 50 \\
\mathrm{~mm} / \text { dia }\end{array}$ & dias \\
\hline PRCPTOT & Precipitação total anual nos dias úmidos & $\begin{array}{l}\text { Precipitação total anual nos } \\
\text { dias úmidos }(R R \geq 1 \mathrm{~mm})\end{array}$ & $\mathrm{mm}$ \\
\hline SDII & Índice simples de intensidade diária & $\begin{array}{l}\text { Quantidade diária de } \\
\text { precipitação nos dias úmidos }\end{array}$ & $\mathrm{mm}$ \\
\hline
\end{tabular}

Tabela 4. Tendência temporal dos índices de extremos climáticos dependentes da precipitação para o estado do Piauí. Os valores em negrito correspondem ao nível de significância estatística de $10 \%(p \leq 0,10)$.

\begin{tabular}{lccccccccccc}
\hline \multicolumn{1}{c}{ Estações } & DCS & DCU & PRCPTOT & R10mm & R20mm & R95p & R99p & R50mm & Rx1dia & Rx5dias & SDII \\
\hline Bom J. do Piauí & $\mathbf{1 , 5 7}$ & $-0,03$ & $\mathbf{- 1 0 , 9 2}$ & $\mathbf{- 0 , 3 4}$ & $-0,16$ & $-3,35$ & $-1,36$ & $-0,05$ & $-0,36$ & $-0,46$ & 0,02 \\
Caldeirão & 1,36 & $-0,29$ & 6,31 & $-0,02$ & 0,23 & 7,86 & $\mathbf{6 , 1 2}$ & 0,14 & 1,54 & $\mathbf{2 , 9 6}$ & $\mathbf{0 , 1 6}$ \\
Esperantina & 0,10 & $-0,02$ & $-4,21$ & $-0,26$ & $-0,14$ & 1,70 & 0,87 & $-0,08$ & 0,63 & 0,69 & 0,02 \\
Floriano & $\mathbf{1 , 4 1}$ & $-0,01$ & $-6,55$ & $-0,06$ & $-0,08$ & $-4,25$ & $-1,59$ & $\mathbf{- 0 , 1 5}$ & $\mathbf{- 1 , 1 6}$ & $-0,13$ & $-0,02$ \\
Luzilandia & $-0,02$ & $-0,12$ & $-9,57$ & $-0,48$ & $-0,23$ & $-1,85$ & $-2,32$ & $-0,02$ & 0,28 & 1,15 & 0,00 \\
M. dos Cavalos & 1,63 & 0,01 & 4,29 & 0,15 & 0,16 & 3,01 & $-1,71$ & 0,01 & - & - & 0,02 \\
Parnaíba & 0,33 & 0,03 & $-28,56$ & $-0,56$ & $-0,42$ & $-18,60$ & $-5,76$ & $-0,27$ & $-1,44$ & $-2,37$ & $\mathbf{- 0 , 2 0}$ \\
Picos & 0,31 & $\mathbf{0 , 0 6}$ & $-1,42$ & $-0,02$ & $-0,04$ & $-0,77$ & $-0,27$ & 0,00 & $-0,02$ & 0,01 & 0,00 \\
Piriri & 0,18 & 0,16 & 11,96 & 0,27 & $\mathbf{0 , 3 1}$ & 5,38 & 4,51 & 0,05 & 1,00 & 0,71 & 0,05 \\
São J. do Piauí & 1,44 & 0,04 & $-6,38$ & $-0,17$ & $\mathbf{- 0 , 1 8}$ & $-0,32$ & $-0,18$ & 0,00 & $-0,23$ & 1,24 & $-0,01$ \\
Teresina & 0,36 & $-0,01$ & $-6,19$ & $-0,16$ & $-0,15$ & 1,70 & $-1,73$ & $\mathbf{- 0 , 1 4}$ & $-0,23$ & $-0,08$ & $-0,03$ \\
V. do Gurguéia & 0,22 & $-0,04$ & $-3,71$ & $-0,17$ & 0,00 & 0,94 & 2,82 & $-0,06$ & 0,55 & 0,00 & 0,11 \\
Caracol & $\mathbf{1 , 9 4}$ & $-0,03$ & $-0,72$ & $-0,12$ & $-0,07$ & $-0,31$ & $-0,82$ & $-0,02$ & $-0,22$ & $-0,55$ & $-0,01$ \\
\hline
\end{tabular}


exigindo, portanto que os dados provenham de duas populações normais. Como o estado do Piauí possui uma diferença climática bem acentuada à medida que avança para o sul, o primeiro passo foi separar as estações que faziam parte do norte e do sul do Estado, a partir disso foi realizada a média anual de cada índice para as estações do norte e do sul. Os índices foram correlacionados individualmente com a anomalia de TSM de cada área estudada. A significância estatística foi obtida através do teste $t$-Student aplicada a uma série que apresenta graus de liberdade $(\mathrm{N})$ igual a 34, tendo um $\mathrm{r}_{\text {crítico }}=0,286(\mathrm{p} \leq 0,05)$, correspondentes aos 34 anos da série histórica de dados analisados.

\section{Resultados e discussão}

\section{Classificação das imagens}

Como resultado de todo o processamento digital das imagens foi obtido os mapas de uso e ocupação das terras para os dois períodos estudados e estes por sua vez podem ser visualizadas nas Figuras $2 \mathrm{a}$ e $2 \mathrm{~b}$. Os mapas obtidos reve- laram que a porção central do Piauí é que apresenta maior percentual de solo exposto e isto é justificável, pois esta área está geograficamente sujeita ao regime pluviométrico do semiárido, além disso, tal região apresentou os menores valores de NDVI, indicando vegetação esparsa e presença de solo exposto. A pouca pluviometria nesta região deve-se aos sistemas meteorológicos que atuam na região assim como sua fisiografia justificada por sua localização. Guedes et al. (2010) explanam que os menores valores de precipitação no Piauí estão na parte central do Estado, região semiárida e a topografia ajuda nessa configuração, visto que a parte norte é mais baixa e favorece a atuação de sistemas meteorológicos de grande escala. Já a parte central e sul do Estado, provavelmente sofre influência de sistemas de escala local como chuvas orográficas.

Verificou-se que as classes de uso do solo "Mata" e "Solo exposto" diminuíram na região, porém o que chama atenção é como a classe da "Cultura" aumentou e, consequentemente, devido à necessidade de irrigação a quantidade de água também aumentou proporcionalmente, tal-

a)
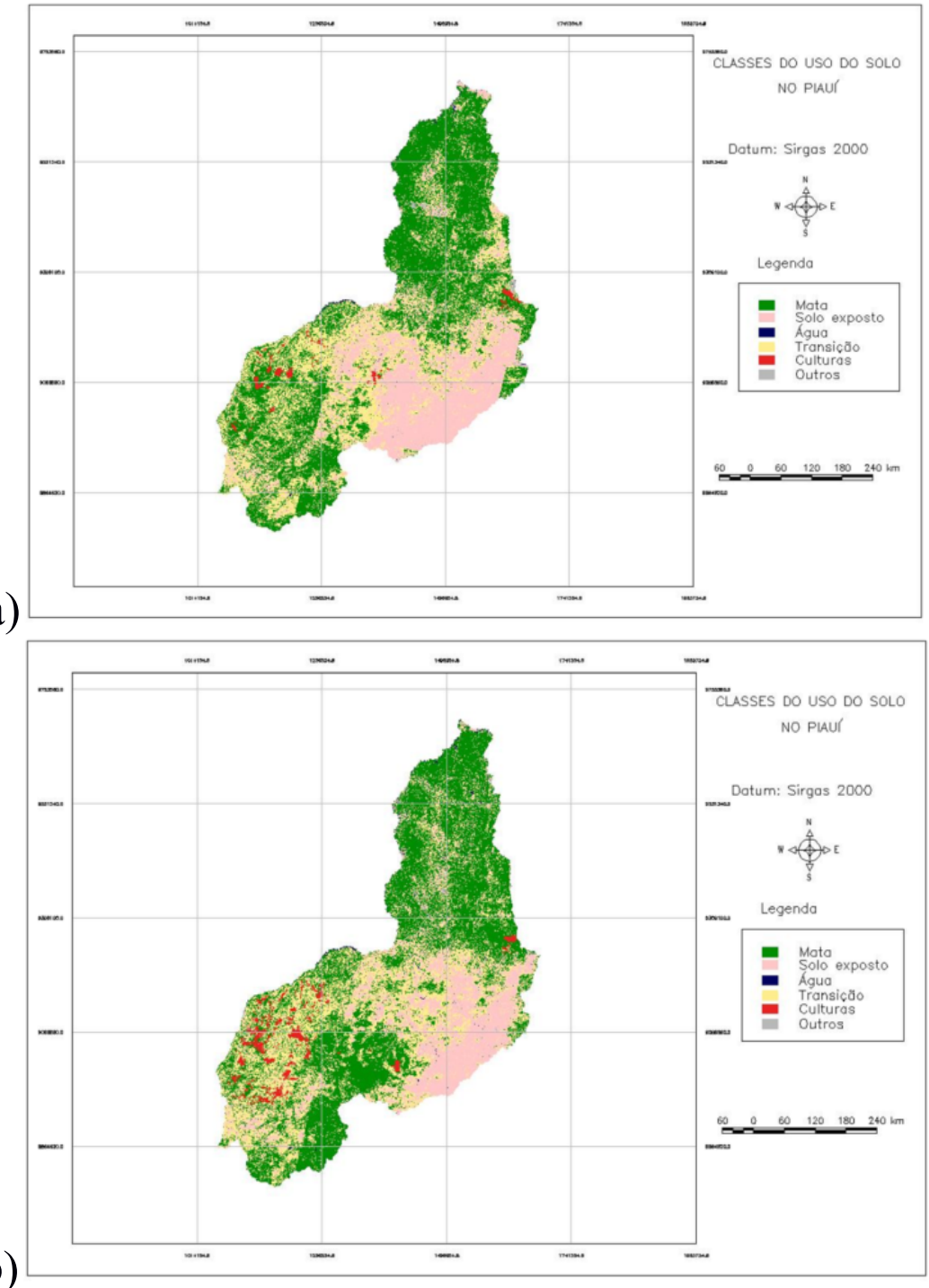

Figura 2. Mapa de uso e ocupação das terras do Estado do Piauí nos anos de a) 1990 e b) 2000. 
vez, oriunda da construção de reservatórios. Os alvos que ficaram confusos foram inseridos na classe "Outros".

\section{Índices de mudanças climáticas}

A Tabela 4 apresenta os valores das tendências da série histórica estudada (período de 1971 a 2010) dos índices de extremos climáticos dependentes da precipitação, de todas as 13 estações. Consideraram-se tendências estatisticamente significativas aquelas em que o valor $\mathrm{p}$ foi inferior ou igual a $0,10(p \leq 0,10)$, os referidos valores estão em negrito na Tabela 4. As estações de Esperantina, Luzilândia, Morro dos Cavalos e Vale do Gurguéia não apresentaram tendências estatisticamente significativas. Foram verificadas tendências estatisticamente significativas nos índices de extremos climáticos DCS $(p=0,016)$, PRCPTOT $(p=0,015)$ e R10mm ( $p=0,016)$ para a localidade de Bom Jesus do Piauí. Na localidade Caldeirão foram observadas tendências estatisticamente significativas nos índices R99p ( $p=0,100)$, $\operatorname{Rx} 5$ dias $(p=0,08)$ e SDII $(p=0,034)$. Em Floriano as referidas tendências foram para os índices DCS $(p=0,009)$, R50mm $(p=0,062)$ e Rx1dia $(p=0,100)$. Em Parnaíba, Picos, Piriri, São Joao do Piauí, Teresina e Caracol apenas os índices SDII ( $p=0,086)$, DCU $(p=0,096), R 20 \mathrm{~mm}(p=0,068), R 20 \mathrm{~mm}$ $(p=0,078)$, R50mm $(p=0,014)$ e DCS $(p=0,096)$, respetivamente, apresentaram tendências estatisticamente significativas.

O índice dias consecutivos secos (DCS) apresenta tendência positiva ao longo do Estado, porém os valores mais altos desse índice estão na porção sul e leste do Piauí. Isso é justificável por essa região sofrer efeitos de sistemas locais tais como aglomerados convectivos e linhas de instabilidade. Concomitante a este resultado está o mapa de uso e ocupação das terras que apresentou para as mesmas porções as maiores áreas de solo exposto. As estações que apresentam significância estatística para este índice são 1 , 4 e 13 que correspondem a Bom Jesus do Piauí, Floriano e Caracol (Figura 3a), respectivamente. Por outro lado o índice dias consecutivos chuvosos ou úmidos (DCU) apresentou configuração inversa, isto é, observa-se valores altos de tendências na parte norte diminuindo em direção ao sul do Estado. Isto ocorre pela influência do clima semiárido na parte sul do Estado (Guedes et al, 2010) (Figura 3b). Ressalta-se ainda que este índice apresenta também um núcleo de tendência negativa no norte do Estado, possivelmente, é devido a eventos de grande escala e por fatores locais. Somente a estação de Picos apresentou significância estatística para este índice.

O padrão das tendências para os índices R10mm e PRCPTOT são similares, isto é, observa-se um decaimento dos índices em todo o Estado do Piauí (Figuras 3c e 3d). A precipitação total anual (PRCPTOT) apresenta, para toda a região estudada, uma variação de $-20 \mathrm{~mm} / \mathrm{ano}$, no extremo norte do Estado, a $-10 \mathrm{~mm} / \mathrm{ano}$, na parte sul (Figura 3c). Isto significa que os totais anuais de chuvas no estado de Piauí, estão diminuindo. O número de dias do ano com precipitação maior que $10 \mathrm{~mm}$ (R10mm) apresenta uma redução em quase todo Estado de Piauí. É possível observar tendências negativas do norte ao sul do Estado (Figura 3d). Salienta-se, ainda, que a frequência de chuvas acima de $10 \mathrm{~mm}$ diminuiu, para estes dois índices apenas a estação 1 (Bom Jesus do Piauí) teve significância estatística. Os resultados corroboram com Guedes et al. (2010).

A tendência de número de dias com precipitação maior que $20 \mathrm{~mm}$ (R20mm) (Figura 4a) mostra valores negativos ao longo do Estado. As estações que tiveram significância estatística para este índice foram 9 e 10 (Piriri e São João do Piauí). O número de dias com precipitação superior a 50 $\mathrm{mm}$ (R50mm) apresentou tendência negativa ao longo do Estado apresentando um pequeno crescimento na área que estão situadas as estações 2 e 9 (Caldeirão e Piriri), ou seja, estas duas estações apresentam um aumento de eventos de precipitação extrema, iguais ou superiores a $50 \mathrm{~mm}$, precipitações dessas magnitudes podem ocasionar inundações e alagamentos, tais eventos causam consequências em curto e longo prazo, através de impactos diretos na agricultura e pecuária, nos recursos hídricos, na infraestrutura urbana, como também nos seres humanos, afetando o bem-estar social (Changnon, 1996). Para este índice as estações que tiveram significância estatística foram 4 e 11 (Floriano e Teresina) (Figura 3b).

O padrão de tendência anual da maior precipitação diária em um dia (R1xdia) apresenta redução em quase todo o Estado do Piauí, a estação que teve significância estatística para este índice foi a 4 (Floriano) (Figura 4c). Por outro lado, a distribuição espacial do índice da maior precipitação em cinco dias consecutivos em um ano (Rx5dias) apresentou tendência positiva em quase todo o Estado, tendo tendência negativa apenas no extremo norte e extremo sul do Estado do Piauí, isto é, a tendência do índice Rx5dias e maior no Estado quando comparado com o índice Rx1dia. A estação 2 (caldeirão) foi a que teve significância estatística para este índice (Figura $4 \mathrm{~d}$ ).

As Figuras 5a e 5b mostram as distribuições espaciais dos índices R95p (soma total da precipitação em um ano de $5 \%$ dos dias mais chuvosos) e R99p (soma total da precipitação em um ano de $1 \%$ dos dias mais chuvosos) para o Estado do Piauí. Ambas apresentaram tendências negativas na porção sul e no extremo norte do Estado, para o índice R99p apenas a estação 2 (caldeirão) se mostrou estatisticamente significativa (Figura 5b). O índice SDII apresentou tendências positivas na maior parte do Estado e tendências negativas apenas no litoral (Figura 5c). O resultado deste índice corrobora com os índices DCU e PRCPTOT onde 

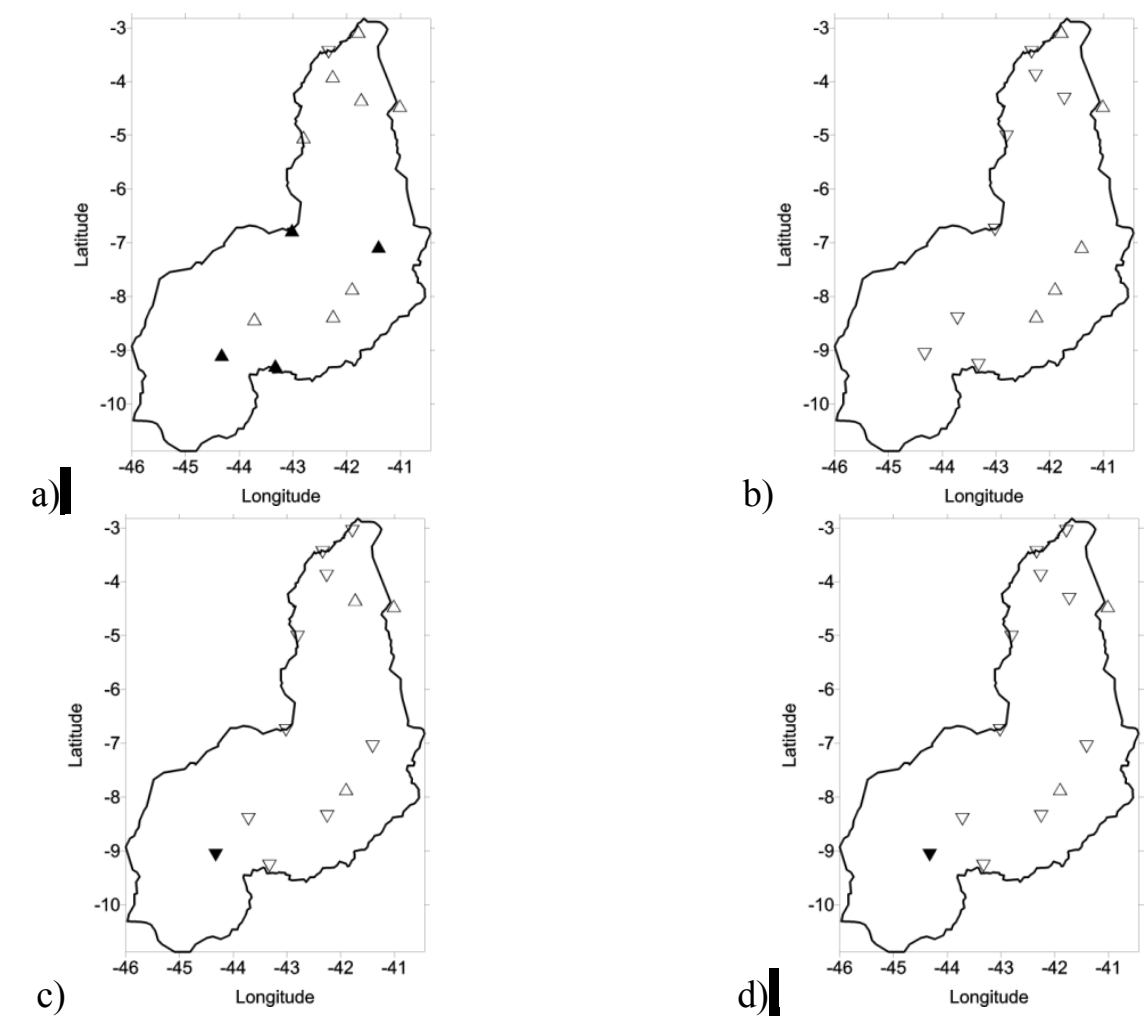

Figura 3. Distribuição espacial da tendência dos índices: a) dias consecutivos secos (DCS) (dias/ano), b) dias consecutivos úmidos (DCU) (dias/ano), c) total anual de precipitação (PRCPTOT) (mm/ano) e d) número de dias com chuvas superiores a $10 \mathrm{~mm}$ (R10mm) (dia/ano) para o estado de Piauí no período de 1971 a 2010.

também foi verificada tendência negativa no litoral do Estado. O índice R95p foi o único que não teve significância estatística em nenhuma das estações estudadas (Figura 5a).

\section{Correlações entre os índices de extremos climáticos e as anomalias de TSM dos Oceanos Tropicais}

Santos e Manzi (2011) afirmam que a variabilidade do clima do NEB está associada a padrões de variação em escala planetária, que estão associados ao comportamento dos oceanos Atlântico e Pacífico. Kayano et al. (2009) pesquisaram as relações entre a precipitação na América do Sul e os índices de TSM dos oceanos tropicais, por meio de análise de correlação para o período de 1948-1976 e 1977-2002. Os resultados mostraram que a relação entre o El Niño (La Niña) e a precipitação é negativa (positiva) para o nordeste da América do Sul e positiva (negativa) para o sul e o sudeste do continente. Como as características climáticas das partes norte e sul do Piauí possuem diferenças bem acentuadas, verificou-se que seria necessário dividir as estações em duas partes (norte e sul). A Tabela 5 apresenta as correlações entre os índices de extremos climáticos dependentes da precipitação diária para a parte norte do Piauí e as anomalias de TSM nos Oceanos Pacífico (Niño 1+2, Niño 3 Niño 3.4 e Niño 4) e Atlântico (TNAI e TSAI). Pode-se verificar que as anomalias de TSM nas regiões de Niño 3, Niño 3.4 e Niño 4 apresentam correlação negativa, com significância estatística, para os índices DCU, PRCPTOT, R10mm, R20mm, R95p, R99p, R50mm, Rx5dias e SDII mostrando que quando a TSM diminui (La Niña) ocorre um aumento de todos os índices anteriormente citados, indicando intensificação dos eventos extremos e do total anual de precipitação sobre a região estudada. Entretanto, a região Niño $1+2$ apresentou significância estatística apenas para os índices PRCPTOT, R10mm, R20 mm e R50mm.

$\mathrm{O}$ índice que representa as anomalias de TSM no Oceano Atlântico Norte (TNAI) não mostrou correlações com significância estatística com nenhum dos índices estudados. Em contrapartida, o índice TSAI apresentou correlação positiva (com significância estatística) para os índices $\mathrm{DCU}, \mathrm{R} 10 \mathrm{~mm}$ e R20mm, indicando que o aquecimento do Oceano Atlântico Tropical Sul induz o deslocamento da Zona de Convergência Intertropical (ZCIT) mais ao sul do Equador terrestre levando a um aumento dos dias consecutivos úmidos e dos eventos de precipitação acima de 10 e $20 \mathrm{~mm}$ sobre a parte norte do Piauí.

Na parte sul do Piauí foi possível observar que no geral as anomalias de TSM não influenciam muito os extremos de precipitação, é possível verificar isso através da Tabela 6 que apresenta as correlações entre os índices de extremos climáticos dependentes da precipitação diária para a parte sul do Piauí e as anomalias de TSM nos Oceanos Pacífico e Atlântico. Apenas as regiões de Niño 3, TNAI e 

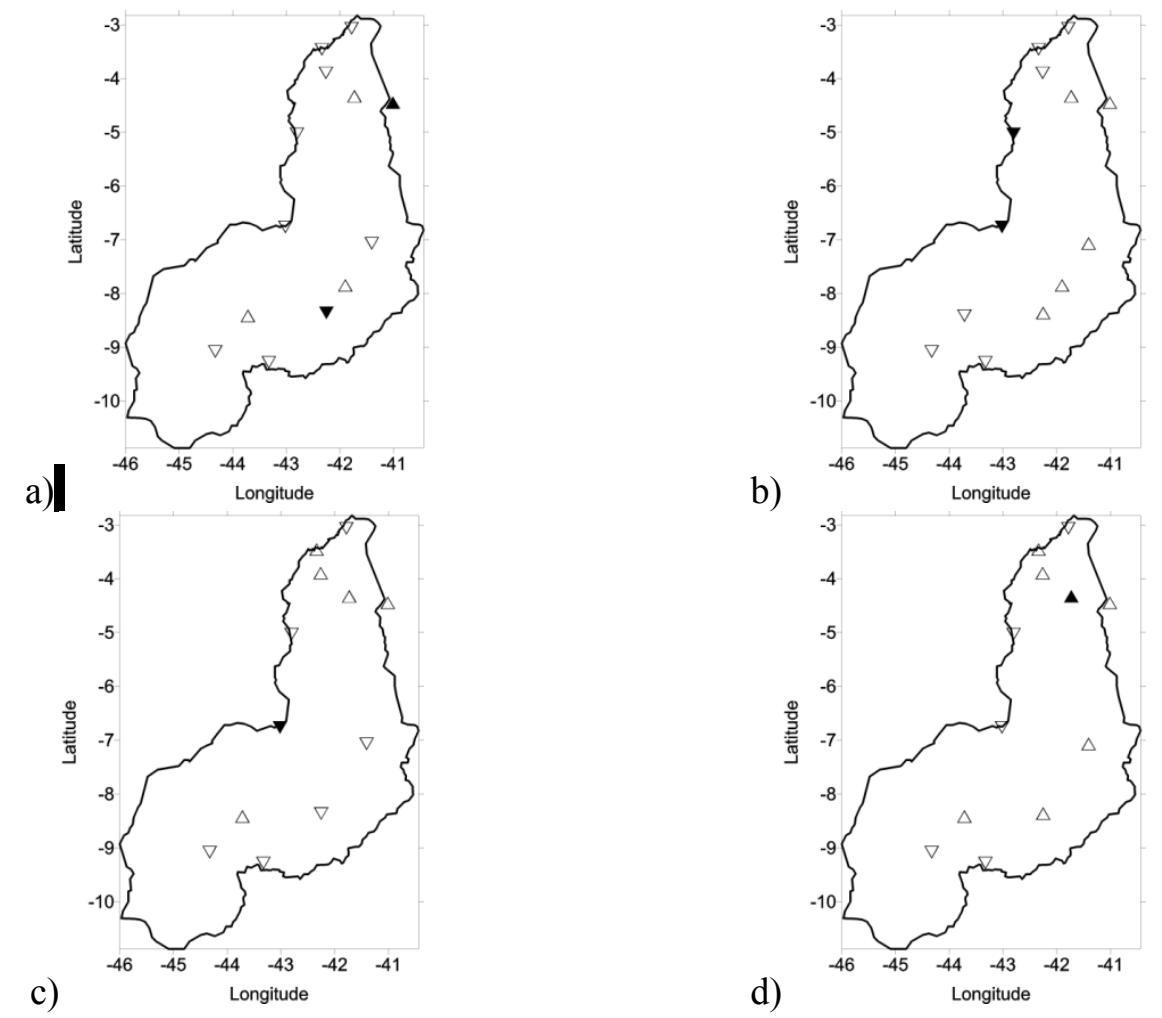

Figura 4. Distribuição espacial da tendência dos índices: a) número de dias com chuvas superiores a $20 \mathrm{~mm}$ (R20mm) (dia/ano), b) número de dias com chuvas superiores a $50 \mathrm{~mm}$ (R50mm) (dia/ano), c) quantidade máxima de precipitação em um dia (Rx1dia) (mm) e d) quantidade máxima de precipitação em 5 dias consecutivos (Rx5dias) (mm) para o estado de Piauí no período de 1971 a 2010.

a)

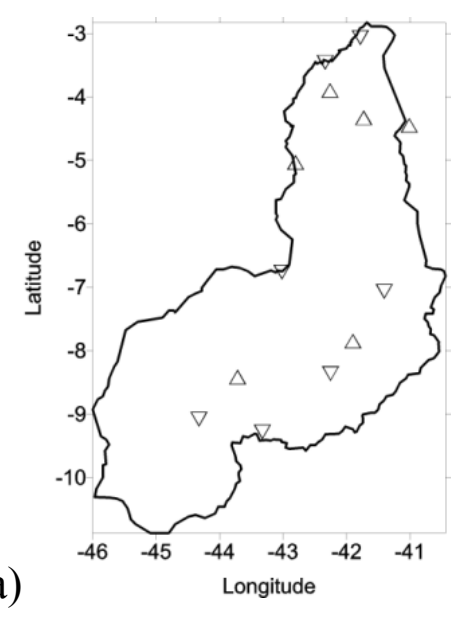

b)

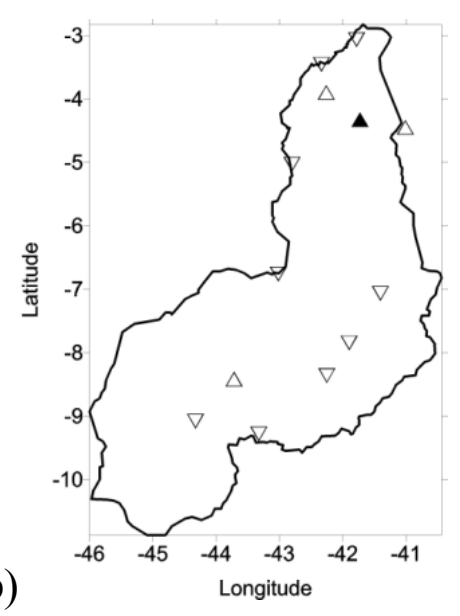

c)

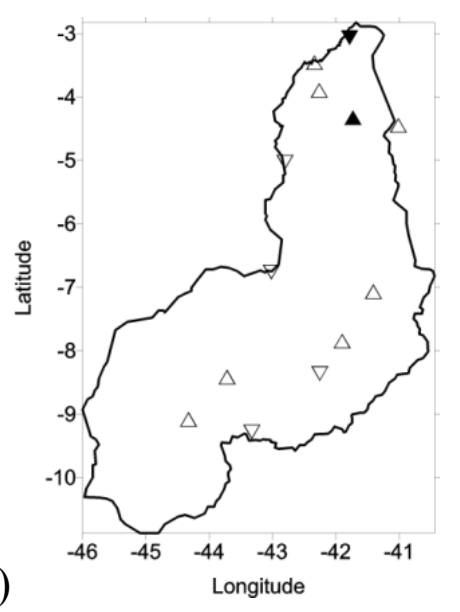

Figura 5. Distribuição espacial da tendência dos índices: a) R99p (mm/ano), b) R95p (mm/ano) e c) SDII (mm/ano) no estado de Piauí.

TSAI apresentaram índices com significância estatística. Sugere-se que a parte sul do Estado esteja mais sujeita a precipitações oriundas de sistemas locais.

\section{Conclusões}

A análise dos índices anuais de extremos climáticos oriundos de dados de precipitação diária indica uma diminuição da precipitação total anual no Estado do Piaui. Em geral, os índices R10mm, R20mm e 50mm apresentaram tendência negativa ao longo do Estado. Os índices DCS e DCU apresentaram padrão inverso de tendências, onde o DCS foi maior na parte sul e o DCU maior na parte norte, podendo ser visualizado também nos mapas de uso e ocupação das terras, visto que a parte norte se apresentou mais vegetada enquanto a parte central e sul do Estado apresentou maior quantidade de solo exposto.

Verificou-se que o regime de chuvas na porção norte 
Tabela 5. Correlações entre os índices de extremos climáticos dependentes da precipitação diária para a parte norte do Piauí e as anomalias de TSM nos Oceanos Pacífico e Atlântico. Os valores em negrito correspondem ao nível de significância de 5\% ( $\leq 0,05)$.

\begin{tabular}{lccccccccccc}
\hline & DCS & DCU & PRCPTOT & R10mm & R20mm & R95p & R99p & R50mm & Rx1dia & Rx5dias & SDII \\
\hline Niño 1+2 & 0,101 & $-0,234$ & $\mathbf{- 0 , 4 2 7}$ & $\mathbf{- 0 , 4 2 1}$ & $\mathbf{- 0 , 4 4 6}$ & $-0,198$ & $-0,054$ & $\mathbf{- 0 , 4 2 1}$ & $-0,192$ & $-0,173$ & $-0,29$ \\
Niño 3 & 0,113 & $\mathbf{- 0 , 3 1 5}$ & $\mathbf{- 0 , 4 2 6}$ & $\mathbf{- 0 , 3 3 3}$ & $\mathbf{- 0 , 3 1 7}$ & $\mathbf{- 0 , 4 6 9}$ & $\mathbf{- 0 , 3 5}$ & $\mathbf{- 0 , 3 7 4}$ & $-0,234$ & $\mathbf{- 0 , 4 2 2}$ & $\mathbf{- 0 , 3 8 8}$ \\
Niño 3.4 & 0,125 & $\mathbf{- 0 , 3 9 2}$ & $\mathbf{- 0 , 4 6 6}$ & $\mathbf{- 0 , 3 7 1}$ & $\mathbf{- 0 , 3 4 5}$ & $\mathbf{- 0 , 5 2 1}$ & $\mathbf{- 0 , 3 4 5}$ & $\mathbf{- 0 , 4 0 6}$ & $-0,179$ & $\mathbf{- 0 , 4 2 7}$ & $\mathbf{- 0 , 3 6 8}$ \\
Niño 4 & 0,083 & $\mathbf{- 0 , 4 3 2}$ & $\mathbf{- 0 , 5 0 6}$ & $\mathbf{- 0 , 3 9 6}$ & $\mathbf{- 0 , 3 8}$ & $\mathbf{- 0 , 5 5 8}$ & $\mathbf{- 0 , 3 3 5}$ & $\mathbf{- 0 , 4 5 4}$ & $-0,121$ & $\mathbf{- 0 , 3 9 6}$ & $\mathbf{- 0 , 3 6 4}$ \\
TNAI & 0,166 & $-0,18$ & $-0,251$ & $-0,222$ & $-0,167$ & $-0,174$ & $-0,146$ & $-0,143$ & $-0,055$ & $-0,258$ & 0,029 \\
TSAI & $-0,193$ & $\mathbf{0 , 3 2 4}$ & 0,238 & $\mathbf{0 , 3 6 7}$ & $\mathbf{0 , 3 1 8}$ & $-0,053$ & $-0,127$ & 0,178 & $-0,128$ & $-0,24$ & $-0,186$ \\
\hline
\end{tabular}

Tabela 6. Correlações entre os índices de extremos climáticos dependentes da precipitação diária para a parte sul do Piauí e as anomalias de TSM nos Oceanos Pacífico e Atlântico. Os valores em negrito correspondem ao nível de significância de $5 \%(p \leq 0,05)$.

\begin{tabular}{lccccccccccc}
\hline & DCS & DCU & PRCPTOT & R10mm & R20mm & R95p & R99p & R50mm & Rx1dia & Rx5dias & SDII \\
\hline Niño 1+2 & 0,112 & $-0,096$ & $-0,161$ & $-0,116$ & $-0,148$ & $-0,003$ & 0,036 & $-0,074$ & 0,067 & 0,145 & 0,16 \\
Niño 3 & 0,076 & $-0,262$ & $\mathbf{- 0 , 3 0 6}$ & $-0,239$ & $-0,238$ & $-0,106$ & $-0,107$ & $-0,191$ & $-0,128$ & $-0,053$ & 0,069 \\
Niño 3.4 & 0,059 & $-0,263$ & $-0,257$ & $-0,188$ & $-0,159$ & $-0,052$ & $-0,061$ & $-0,118$ & $-0,116$ & 0,031 & 0,19 \\
Niño 4 & 0,158 & $-0,215$ & $-0,265$ & $-0,205$ & $-0,111$ & $-0,052$ & $-0,021$ & $-0,093$ & $-0,137$ & 0,087 & $\mathbf{0 , 3 1 7}$ \\
TNAI & 0,213 & $-0,016$ & $\mathbf{- 0 , 2 8 9}$ & $\mathbf{- 0 , 2 9 2}$ & $-0,217$ & $-0,078$ & 0,089 & $-0,187$ & $-0,154$ & $-0,014$ & 0,052 \\
TSAI & $-0,01$ & 0,106 & $-0,033$ & 0,126 & $-0,134$ & $\mathbf{- 0 , 3 6 6}$ & $-0,157$ & $\mathbf{- 0 , 2 9 7}$ & $-0,117$ & $-0,268$ & $-0,282$ \\
\hline
\end{tabular}

do Estado sofre influência das anomalias de TSM do Pacífico e do Atlântico. Os índices que representam os extremos de chuva apresentaram correlações de significância estatística com as regiões de Niño 1+2 e Niño 3, Niño 3.4, Niño 4 no Pacífico e com a região do Atlântico Sul Tropical. Evidenciando a forte influência que a atuação do ENOS e do Dipolo do Atlântico têm sobre o clima do Piauí. Porém, a parte sul do Estado apresentou poucos resultados com significância estatística sugerindo que o regime de chuvas nessa região tenha como fator influenciador os sistemas locais.

\section{Agradecimentos}

O primeiro autor agradece ao Conselho Nacional de Desenvolvimento Científico e Tecnológico (CNPq) pela Bolsa de Produtividade em Pesquisa, assim como à Coordenação de Aperfeiçoamento de Pessoal de Nível Superior (CAPES) pelo financiamento do Projeto de Pesquisa intitulado "Análise e Previsão dos Fenômenos Hidrometeorológicos Intensos do Leste do Nordeste Brasileiro" - Edital Pró-Alertas no 24/2014 (Processo nº 88887.091737/2014-01).

\section{Referências}

ALEXANDER, L.V.; ZHANG, X.; PETERSON, T.C.; CAESAR, J.; GLEASON, B.; et al. Global observed changes in daily climate extremes of temperature and precipitation. Journal of Geophysical Research, v. 111, D05109, 2006. http://dx.doi.org/10.1029/2005JD006290.
ALMEIDA-FILHO, R.; CARVALHO, C. M. Mapping land degradation in the Gilbués region, northeastern Brazil, using Landsat TM images. International Journal of Remote Sensing, v. 31, p. 1087-1094, 2010.

CHANGNON AS. 1996. The Great Flood of 1993: Causes, Impacts, and Responses. Westview Press: Boulder; 321pp., 1996.

GUEDES, R. V. S.; LIMA, F. J. L.; AMANAJÁS, J. C.; BRAGA, C. C.; Análise em componentes principais da precipitação pluvial no estado do Piauí e agrupamento pelo Método de Ward. Revista de Geografia, v. 27, p. 218-233, 2010.

GUERREIRO, M. J. S.; ANDRADE, E. M.; ABREU, I.; LAJINHA, T. Longterm variation of precipitation indices in Ceará State, Northeast Brazil. International Journal of Climatology, v. 33, p. 2929-2939, 2013.

HASTENRATH, S. Exploring the climate problems of Brazil's Nordeste: a review. Climatic Change, v. 112, p.243-251, 2012.

HASTENRATH, S., GREISCHAR, L. Further Work on the Prediction of Northeast Brazil Rainfall Anomalies. Journal of Climate, v. 6, p. 743-758, 1993.

HAYLOCK, M. R.; PETERSON, T. C.; ALVES, L. M.; AMBRIZZI, T.; ANUNCIAÇÃO, T. M. T.; BAEZ, J.; BARROS, V. R.; BERLATO, M. A.; BIDEGAIN, M.; CORONEL, G.; GARCIA, V. J.; GRIMM, A. M.; KAROLY, D.; MARENGO, J. A.; MARINO, M. B.; MONCUNILL, D. F.; NECHET, D.; QUINTANA, J.; REBELLO, E.; RUSTICUCCI, M.; SANTOS, J. L.; TREBEJO, I.; VINCENT, L. A.; Trends in total and extreme South American rainfall 1960-2000 and links with sea surface temperature. Journal of Climate, v. 19, p. 1490-1512, 2006.

KAYANO, M. T., OLIVEIRA, C. P., ANDREOLI, R.V. Interannual relations between South American rainfall and tropical sea surface temperature anomalies before and after 1976. International Journal of Climatology, v. 29, p.1439-1448, 2009.

KLEIN TANK, A.M.G.; PETERSON, T.C.; QUADIR, D.A.; DORJI, S.; ZOU, X.; et al. Changes in daily temperature and precipitation extremes in Central and South Asia. Journal of Geophysical Research, v. 111, D16105, 2006. http://dx.doi.org/10.1029/2005JD006316.

MENEZES, H. E. A.; BRITO, J. I. B.; SANTOS, C. A. C.; SILVA, L. L. A relação entre a temperatura da superfície dos oceanos tropicais e a duração dos veranicos no estado da Paraíba. Revista Brasileira de Meteorologia, v. 23, p.152-161, 2008. 
NEW, M.; HEWITSON, B.; STEPHENSON, D.B.; et al. Evidence of trends in daily climate extremes over southern and west Africa. Journal of Geophysical Research, v. 111, D14, 2006. http://dx.doi. org/10.1029/2005JD006289.

OYAMA, M. D.; NOBRE, C. A. Climatic Consequences of a Large-Scale Desertification in Northeast Brazil: A GCM Simulation Study. Journal of Climate, v. 17, p. 3203-3212, 2004.

SANTOS, C. A. C., BRITO, J. I. B. Análise dos índices de extremos para o semi-árido do Brasil e suas relações com TSM e IVDN. Revista Brasileira de Meteorologia, v. 22, p. 303-312, 2007.

SANTOS, C. A. C.; MANZI, A. O. Eventos extremos de precipitação no estado do Ceará e suas relações com a temperatura dos Oceanos Tropicais. Revista Brasileira de Meteorologia, v. 26, p. 157-165, 2011.

SANTOS, C. A. C.; NEALE, C. M. U.; RAO, T. V. R.; SILVA, B. B. Trends in indices for extremes in daily temperature and precipitation over Utah, USA. International Journal of Climatology, v. 31, p.1813-1822, 2011.

SNEYERS, R. On the Statistical Analysis of Series of Observations. Technical Note No. 143, WMO No. 415 World Meteorological Organization: Geneva. 1990.
UVO, C. B., REPELLI, C. A., ZEBIAK, S. E., KUSHNIR, Y. The Relationships between Tropical Pacific and Atlantic SST and Northeast Brazil Monthly Precipitation. Journal of Climate, v. 11, p. 551-562, 1998.

VILLAFUERTE II, M. Q.; MATSUMOTO, J.; AKASAKA, I.; TAKAHASHI, H. G.; KUBOTA, H.; CINCO, T. A. Long-term trends and variability of rainfall extremes in the Philippines. Atmospheric Research, v. 137, p. 1-13, 2014

VINEY, N. R.; BATES, B. C. It never rains on Sunday: The prevalence and implications of untagged multi-day rainfall accumulations in the Australian high quality data set. International Journal of Climatology, v. 24, p.1171-1192, 2004.

ZHANG, X.; AGUILAR, E.; SENSOY, S.; et al. Trends in Middle East climate extreme indices from 1950 to 2003. Journal of Geophysical Research, v. 110, D22104, 2005. http://dx.doi.org/10.1029/2005JD006181.

ZHANG, X.; HEGERL, G.; ZWIERS, F. W.; KENYON, J.; Avoiding inhomogeneity in percentile-based indices of temperature extremes. Journal of Climate, v. 18, p. 1641-1651, 2005.

ZHANG, X.; YANG, F.; RClinDex (1.0) User Guide. Climate Research Branch Environment Canada: Downsview, Ontario, Canada, 2004.

\section{REFERENCIAÇÃo SANTOS, C. A. C. dos; RAMOS, A. R. D. Avaliação dos eventos extremos de precipitação no estado do Piauí. Agrometeoros, Passo Fundo, v.25, n.1, p.47-57, 2017.}

Declaração: os trabalhos estão sendo publicados nesse número de AGROMETEOROS (v.25, n.1, ago 2017) conforme foram aceitos pelo XX Congresso Brasileiro de Agrometeorologia, realizado de 14 a 18 de agosto de 2017, em Juazeiro, BA e Petrolina, PE, sem revisão editorial adicional da revista. 


\title{
Evaluation of extreme precipitation events in the State of Piauí
}

\author{
Carlos Antonio Costa dos Santos $\left.{ }^{1 *}\right)$ e Anna Raquel D. Ramos ${ }^{2}$ \\ ${ }^{1}$ Professor, Unidade Acadêmica de Ciências Atmosféricas, Centro de Tecnologia em Recursos Naturais, Universidade Federal de Campina Grande - \\ UFCG, Av. Aprígio Veloso, 882, Bairro Universitário, Campina Grande - PB. Email: carlos.santos@ufcg.edu.br \\ ${ }^{2}$ Mestre em Meteorologia, Unidade Acadêmica de Geografia, Centro de Humanidades, Universidade Federal de Campina Grande - UFCG, Av. Aprígio \\ Veloso, 882, Bairro Universitário, Campina Grande - PB. Email: anna.raquelramos@gmail.com \\ ${ }^{(*)}$ Corresponding author
}

ARTICLE INFO

Article history:

Received 16 June 2017

Accepted 10 August 2017

\section{Index terms:}

desertification

El Niño

land use

sea surface temperature

\section{ABSTRACT}

This study examined the spatio-temporal dynamics of climatic extremes indices of daily rainfall in the state of Piauí, correlating them with the land use and cover, and, with sea surface temperature (SST) anomalies of the Atlantic and Pacific Oceans. It was used 34 images of the Landsat 5-TM and daily rainfall data of 13 meteorological stations obtained from the National Institute of Meteorology (INMET). In addition, data of the SST anomalies of the Tropical Oceans for the selected regions have been used. It was observed decrease in annual rainfall in the state of Piauí. It was found that the rainfall in the northern part of the state is influenced by SST anomalies in the Pacific and Atlantic. The indices that represent the extreme rainfall showed correlations with statistical significance, with the regions of Niño $1+2$ and Niño 3, Niño 3.4, Niño 4 in the Pacific and the South Atlantic Tropical region. Therefore, there is a strong influence of ENSO and Atlantic Dipole in the climate of Piauí. However, the southern part of the state had few statistically significant results suggesting that the rainfall in this region may be more influenced by local systems.

(C) 2017 SBAgro. All rights reserved. 